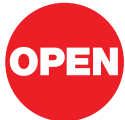

SUBJECT AREAS: TISSUE ENGINEERING MUSCLE STEM CELLS REGENERATION STEM-CELL NICHE

Received

3 December 2012

Accepted

25 February 2013

Published

12 March 2013

Correspondence and requests for materials should be addressed to

A.M. (antonio. musaro@uniromal.it)

* These authors contributed equally to this work.

\section{Generation of eX vivo-vascularized Muscle Engineered Tissue (X-MET)}

\author{
Silvia Carosio ${ }^{*}$, Laura Barberi ${ }^{1 *}$, Emanuele Rizzuto² ${ }^{2}$ Carmine Nicoletti ${ }^{1}$, Zaccaria Del Prete ${ }^{2,3}$ \\ \& Antonio Musarò ${ }^{1,3}$ \\ 'Institute Pasteur Cenci-Bolognetti, DAHFMO-Unit of Histology and Medical Embryology, IIM, Sapienza University of Rome, Italy, \\ ${ }^{2}$ Department of Mechanical and Aerospace Engineering, Sapienza University of Rome, Italy, ${ }^{3}$ Center for Life Nano Science@ \\ Sapienza, Istituto Italiano di Tecnologia, Italy.
}

The object of this study was to develop an in vitro bioengineered three-dimensional vascularized skeletal muscle tissue, named eX-vivo Muscle Engineered Tissue (X-MET). This new tissue contains cells that exhibit the characteristics of differentiated myotubes, with organized contractile machinery, undifferentiated cells, and vascular cells capable of forming "vessel-like" networks. X-MET showed biomechanical properties comparable with that of adult skeletal muscles; thus it more closely mimics the cellular complexity typical of in vivo muscle tissue than myogenic cells cultured in standard monolayer conditions. Transplanted X-MET was able to mimic the activity of the excided EDL muscle, restoring the functionality of the damaged muscle. Our results suggest that $\mathrm{X}-\mathrm{MET}$ is an ideal in vitro 3D muscle model that can be employed to repair muscle defects in vivo and to perform in vitro studies, limiting the use of live animals.

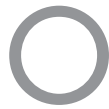

ne of the most ambitious targets of modern medical science is to gain the ability to control and guide the regeneration process of damaged tissues in human body. This desirable and complicated goal arises from the observation that tissues contain stem cells, which are specialized for tissue regeneration. Stem cells can be considered as reserve cells for the organism and are activated when there is a need to repair a portion of damaged tissue.

Cell-based therapies in muscle have been stalled by a number of factors that result in a limited impact of transplanted stem cells on tissue cell replacement. For example, it is difficult to obtain sufficient numbers of autologous cells; injected cells survive poorly; migration of cells from the injection site is minimal; and the surviving population of cells displays rapid senescence. For these reasons, there are to date no satisfactory protocols for therapeutic purposes in the field of muscle regeneration ${ }^{1,2}$. Thus, despite the remarkable potential clinical applications of different stem-cell populations, their use in skeletal muscle is currently hindered by numerous hurdles.

In addressing this problem, outstanding progress has been recently made in tissue engineering technology, with the goal of creating organoids in vitro from cells and cellular scaffolding. Tissue engineering is a novel scientific approach that attempts to mimic neo-organogenesis to "produce" ex-vivo living tissue". This is supported by the fact that stem cells are not an "isolated" population in vivo, but reside within tissue-specific niches that influence their functional behaviour.

Initial clinical experiences with bioengineered tissues have already been reported in skin, cartilage, vascular grafts, bones and several other specialized internal tissues, such as liver and kidney ${ }^{4,5}$.

However, due to its intrinsic complexity, skeletal muscle remains a challenge for in vitro-engineering of tissue. In particular, most of the engineered muscle structures have been obtained employing an artificial scaffold such as matrigel ${ }^{6,7}$, or native or modified collagen ${ }^{8-10}$, limiting the capability to measure and characterize their contractile properties.

The first reported indication that primary skeletal muscle cells could self-organize into a 3-D construct was presented by Strohman and colleagues ${ }^{11}$. Dennis and Kosnik reported the development of cylindrical muscle-like constructs, termed "myooids"12, generated from primary cultures of adult rat myogenic precursor cells and engineered with synthetic tendons.

The objectives of the present study were to generate a vascularized in-vitro 3-dimensional skeletal muscle construct, and to characterize the progenitor cells necessary to form the engineered muscle. The resulting construct was named X-MET, for eX vivo Muscle Engineered Tissue. The final target for our X-MET constructs was to reproduce the morphological and functional properties, showing a close resemblance with the cellular complexity of mature myofibers, both in vitro and in vivo, once transplanted in injured muscles of animal models. 


\section{Results}

Generation of the X-MET and characterization of cell populations participating to its formation. We report the generation of an in vitro engineered muscle-like structure, termed eX vivo Muscle Engineered Tissue (X-MET) obtained from the heterogeneous cell populations that characterize adult skeletal muscles. One of the critical parameter in the context of stem cells and tissue biology is the niche in which they reside. Thus, when taking into account the generation of a new tissue, it is important to consider the way it may regenerate during the normal tissue turnover and create in vitro the conditions that mimic the muscle environment in vivo.

Skeletal muscles are made up of individual components known as myofibers, which are formed from the fusion of myoblasts. Skeletal muscles are also invested with a set of connective tissue sheaths that give a support and protection for the cells and allow them to withstand the contraction forces. Skeletal muscles have also an abundant supply of blood vessels that, together with the endothelial cells, nourishes the tissue and the different cell populations associated with $\mathrm{it}^{13}$. Based on this presupposition, we characterized the best possible culture conditions to generate an X-MET that possesses most of the molecular, morphological and functional properties of a muscle fiber and therefore making it more suitable for an efficient therapeutic approach.

Of note, the heterogeneity of cell populations isolated from skeletal muscle was the prerequisite for the formation of the X-MET, since pure cultures of either satellite cells or fibroblasts failed to generate the X-MET structure (data not shown).

To define the type of progenitor cells associated with muscle niche participating to the generation of X-MET, we used flow cytometry assay. The presence and percentage of myogenic cells has been validated by $\alpha 7$-integrin expression ${ }^{14}$, while precursor cells with myogenic potential have been analyzed by Sca-1 expression ${ }^{15}$. The expression of a specific marker of endothelial cells, namely CD31, was also analyzed ${ }^{16}$. One day after isolation, muscle-derived cells that had adhered to collagen-coated dishes were enzymatically detached from the plates and then analyzed by flow cytometry.

On average, CD31 was expressed by $50 \%$ of total cells (Fig. 1a). Approximately $60 \%$ of $\mathrm{CD} 31^{-}$cells were Sca- $1^{-} / \alpha 7^{+}$representing the myogenic compartment, while about $17 \%$ of $\mathrm{CD} 31^{-}$cells were Sca$1^{+} / \alpha 7^{-}$(Fig. 1a), indicating the presence of interstitial non-muscle cells ${ }^{17}$. This cytofluorimetric profile demonstrates the complexity of skeletal muscle and clearly indicates the different cell precursors which participate in the formation of X-MET.

Plating the heterogeneous cell population isolated from skeletal muscle, namely satellite cells and non-muscle cells resulted in a monolayer consisting of densely packed differentiated myotubes, committed myoblasts and other non myogenic cells, including endothelial cells and fibroblasts, the main producer of extra cellular matrix (ECM) (Fig. 1b). X-MET formation began with peripheral delamination of the cell monolayer, stimulated by gently moving a sterile tip around the peripheral area of the plate (Fig. 1c). This process progressed inward until the entire monolayer had peeled away from the substrate (Fig. 1d).

Once it was delaminated, the monolayer was pinned on a siliconecoated dish using two $0.10 \mathrm{~mm}$ diameter stainless steel minutien pins. Over a period of 2-3 days, the monolayer grew into a selforganized 3D-cylindrical structure (Fig. 1e), containing beating myotubes (Supplementary Video 1).

We also tested the capacity of different combinations of cell populations to generate an X-MET structure. In particular, we observed that a cell culture composed of fibroblasts and satellite cells was able to form a cell monolayer that successfully delaminated from the plate and formed a 3D structure. However, without endothelial cells, the 3D structure did not form the complex and vascularized structure typical of X-MET (see below).
$\mathrm{X}$-MET displays a structural similarity and a molecular complexity typical of in vivo muscle fibers. To verify whether the engineered 3D $\mathrm{X}$-MET has a muscle-like organization, we analyzed the expression of relevant markers of the myogenic program, such as Pax-7, MyoD and myogenin, at different stages of the X-MET formation, which was maintained in differentiation medium (DM): day 0 (the delamination stage, after 5 days in DM), day 5 (corresponding to day 10 in DM) and day 10 (corresponding to day 15 in DM) after X-MET formation. As shown in figure $2 \mathrm{a}$, a marked increase in MyoD transcription was observed at the stage of delamination (day 0), followed by a down regulation at 5 and 10 days. In contrast, Pax-7 and myogenin were expressed at similar levels over the time analysed (Fig. 2a). Notably, real time PCR analysis showed that 3D X-MET structure significantly up-regulated Pax-7 compared to the 2D culture system, whereas MyoD and myogenin were expressed at same levels between 2D and 3D-X-MET structure (data not shown). Altogether, these data indicate that X-MET structure guarantees an ideal environment, including the formation of a basal lamina, for the maintenance of Pax-7 positive undifferentiated satellite-like cells and for the specification of the myogenic identity ${ }^{18}$.

Morphological analysis (Fig. 1b) revealed that myotubes, a differentiated muscle cell types, constitute the predominant cell population within the X-MET. To support this evidence we analysed the expression and sub-cellular localization of myosin heavy chain (MyHC), a molecular marker of differentiated myotubes. Immunofluorescence analysis, performed on transverse sections of X-MET revealed a high expression level of myosin heavy chain ( $\mathrm{MyHC}$ ) (Fig. 2b). In addition, this analysis revealed a longitudinal distribution of parallel myotubes, an essential feature to guarantee the functionality of the construct. Immunofluorescence analysis (Fig. 2b, MyoD and Myogenin panels) revealed also the presence of small percentage of MyHC negative cells which expressed either MyoD or Myogenin, two relevant markers of undifferentiated and committed satellite cells, supporting the data of gene expression analysis (Fig. 2a).

Three layers of connective tissue are part of a muscle in vivo: an outer epimysium, a central perimysium, and an inner endomysium, the delicate connective layer that surrounds the individual skeletal muscle fibers.

To verify whether the X-MET myotubes were surrounded by layers of connective tissue, typical for skeletal muscle, we analysed the expression of laminin, a molecular marker of extracellular matrix (ECM) expressed by endomysium, and ERTR7, a relevant marker of fibroblasts ${ }^{19}$. Immunofluorescence analysis (Fig. 2c, d), revealed that ECM is produced in X-MET and indicates that laminin is correctly organized within the X-MET structure, surrounding the myotubes (Fig. 2d), similarly to that observed in muscle section (Fig. 2c, insert). The production of ECM likely supports the maintenance of the structural integrity of X-MET.

It is known that satellite cells ${ }^{20}$ are scattered between the endomysium and muscle fibers. Light microscopy revealed the presence of round-shaped cells strictly associated with X-MET and localized in an anatomical site typical of satellite cells (Fig. 2e). To support these morphological evidences we analyzed the expression and sub-cellular localization of one of the most relevant marker of this population, namely Pax $-7^{14}$ along with laminin expression. Double immunofluorescence analysis revealed that $\mathrm{Pax}-7$ positive cells localize on the surface of X-MET and are surrounded by laminin (Fig. $2 \mathrm{f}$ and insert). Moreover, we observed the presence of clusters of Pax-7 positive cells (Fig. 2g), similarly to that observed in isolated muscle myofibers ${ }^{21}$. The nature and characteristic of satellite-like cell within the X-MET was further revealed at molecular level by the induction of a coinherited nls-lacZ transgene, driven by the desmin promoter, which is selectively induced in activated and mobilized satellite cells (Fig. 2h $)^{22}$. 

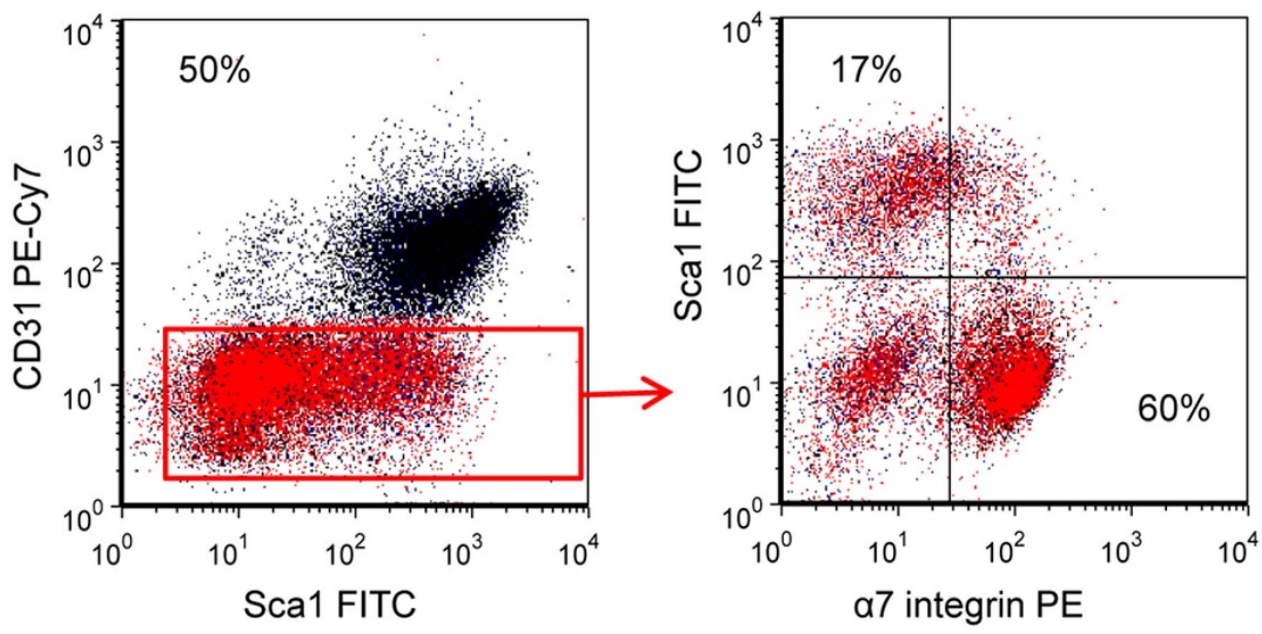

b

C
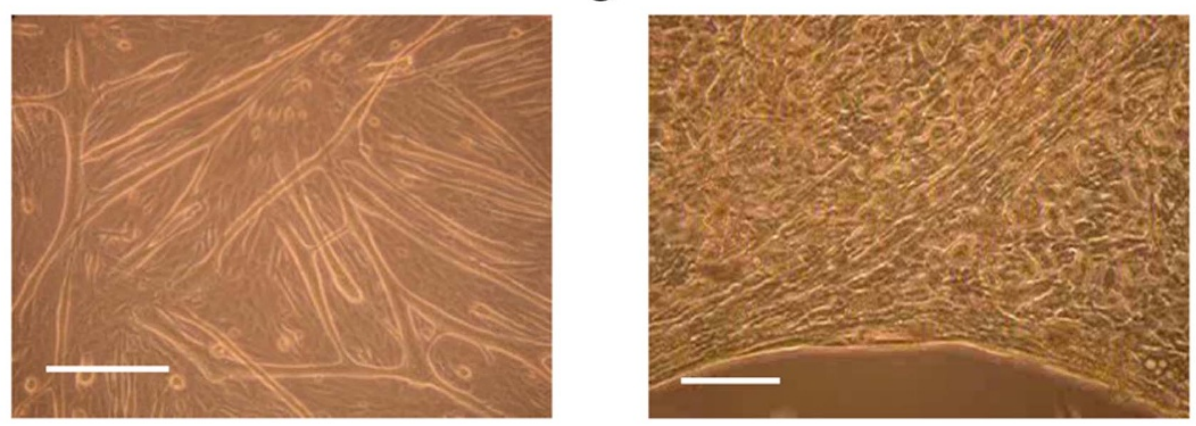

d

e
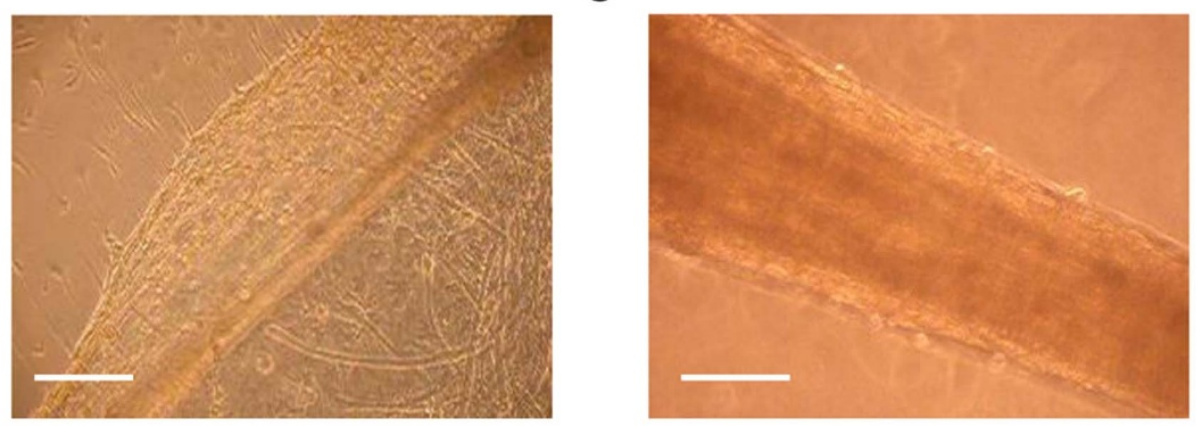

Figure $1 \mid \mathrm{X}$-MET formation and characterization of cell populations participating to the process. (a) Prospective isolation of progenitor populations from skeletal muscle, analysed by flow cytometer. Viable cells were identified based on forward/side scatter, SYTOX ${ }^{\circledR}$ Blue was used to exclude dying or dead cells. Haematopoietic (CD45) were also excluded from analysis. CD31 ${ }^{-}$cells (red area) but not CD31 ${ }^{+}$cells were positive for Sca- 1 and $\alpha 7$ integrin. $60 \%$ of $\mathrm{CD} 31^{-}$cells were Sca- $1^{-} / \alpha 7^{+}$representing the myogenic compartment, while about $17 \%$ of CD $31^{-}$cells were Sca- $1^{+} / \alpha 7^{-}$. (b) Monolayer of differentiated myotubes and committed myoblasts on a substrate of fibroblasts after 5 days in culture. (c) Initial phase of delamination. (d) Delaminated monolayer. (e) Self-organized cylindrical structure obtained 2 days after anchoring the monolayer. Scale bar, $200 \mu \mathrm{m}$.

These data interestingly indicate that the satellite cell niche is being reconstructed in vitro in the X-MET structure.

X-MET is vascularized. Appropriate $3 \mathrm{D}$ tissue structure requiers vascularisation $^{23}$. To support the cytofluorimetric analysis (Fig. 1a) and to investigate the presence and the organization of endothelial cells, we analysed at a morphological and molecular levels the presence of a vascular system within the X-MET structure. Hematoxylin and eosin staining revealed the presence, within the $\mathrm{X}$-MET constructs, of several vessel-like structures with lumenslike (Fig. 3a, black arrows).

Immunofluorescence analysis for CD31 clearly shows the organization of endothelial cells forming a perimeter ring with interior rays which permeate the 3D structure of X-MET (Fig. 3b). Because blood vessels are stabilized by association with smooth muscle cells and because endothelial cells can induce the differentiation of undifferentiated mesenchymal cells into smooth muscle cells, we performed double immunofluorescence analysis for $\alpha$-SMA, a molecular marker of smooth muscle cells, and CD31 (Fig. 3c). Figure 3c shows the organization of smooth muscle cells and endothelial cells into vessellike structures, similarly to that observed in skeletal muscle (Fig. 3c, right panel). Moreover, we examined the expression of key vasculogenic and angiogenic factors, such as CD31, VE-cadherin, vascular endothelial growth factor (VEGF) and its receptor flik1 (Fig. 3d). Real time PCR analysis confirmed that X-MET expresses all of the relevant markers of the vascular structures, and also showed that the 


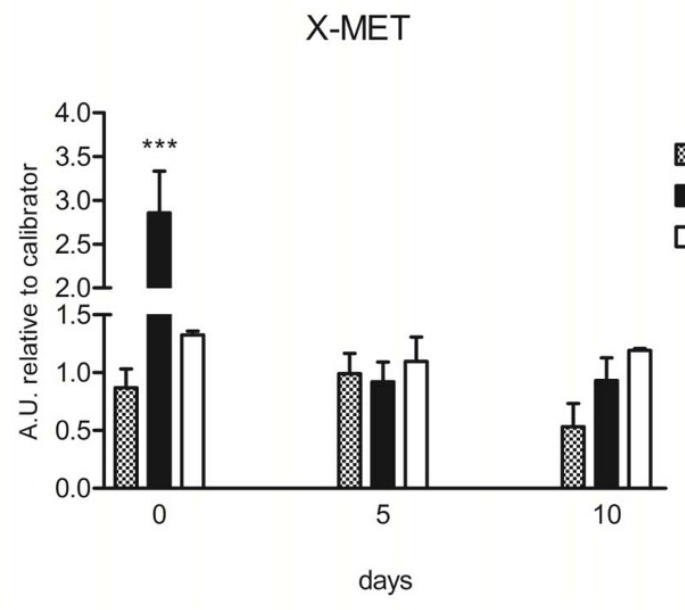

c

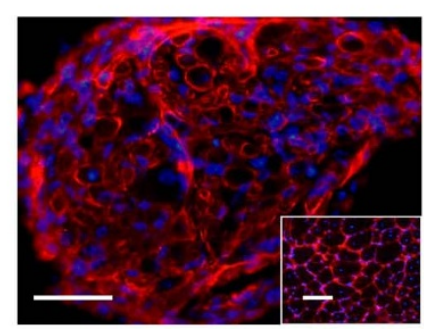

e
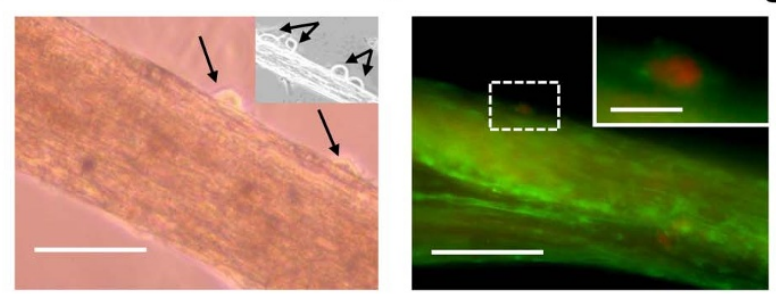

b
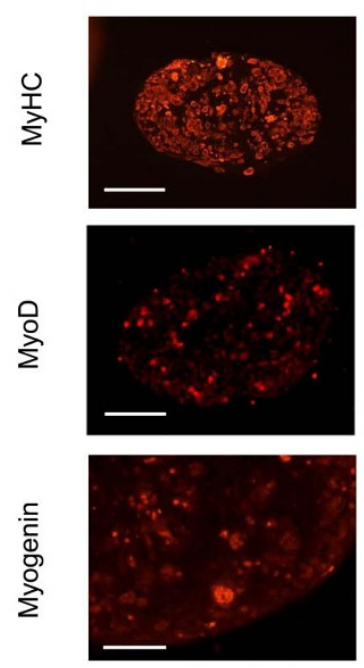

MyHC
Merge
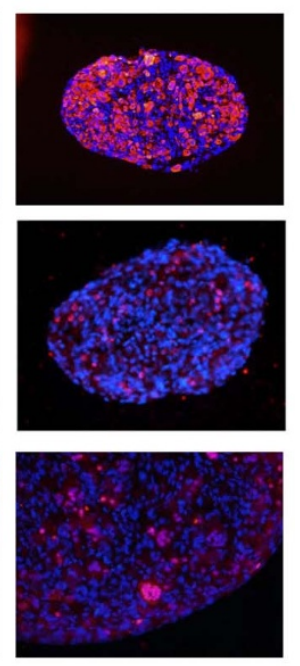

Merge d

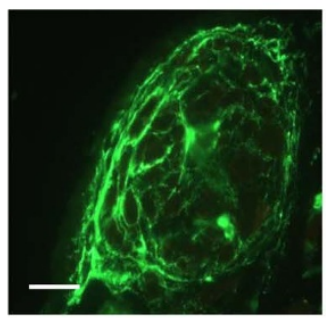

g
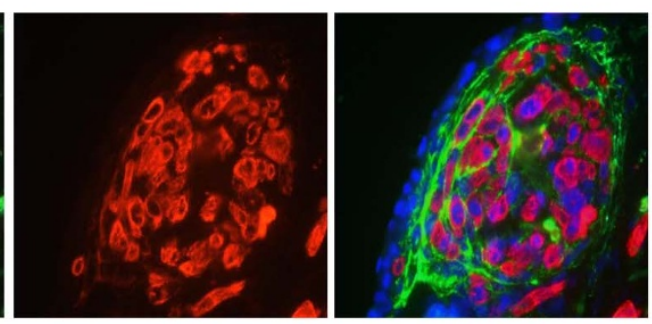

h
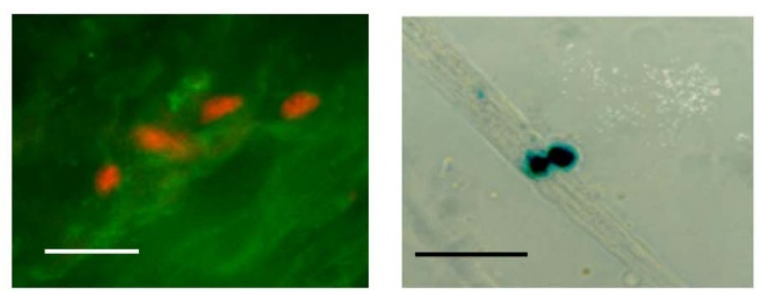

Figure $2 \mid$ Muscle gene expression and morphological organization. (a) Histograms show the expression of relevant markers, measured by qRTPCR, of committed and activated satellite cells during a time course of X-MET formation. At time 0 the X-MET showed a statistically very significant up regulation of $\mathrm{MyoD}$ if compared to the other time points $(* * * \mathrm{p}<0.001)$, while the expression of myogenin remained unchanged over time.

(b) Immunofluorescence analysis for MyHC, MyoD and Myogenin expression on cross sections of X-MET at 10 days in culture. Scale bar, $100 \mu \mathrm{m}$. (c) Immunofluorescence analysis performed on cross sections of X-MET, cultured for 10 days, for laminin expression; insert shows laminin expression in skeletal muscle. Hoechst was used for nuclear staining (blue). Scale bar, $100 \mu \mathrm{m}$. (d) Immunofluorescence analysis performed on cross sections of XMET, cultured for 10 days, for ERTR7 expression and MyHC. Merge image reveals the presence of myotubes positive for MyHC (red) surrounded by connective layers composed by fibroblast positive for ERTR7 (green). Hoechst was used for nuclear staining (blue). Scale bar, $10 \mu \mathrm{m}$. (e) Light microscopy of whole mount X-MET, after 10 days in culture. Light microscopy image revealed the presence of mononucleated cells (black arrows), localized at the typical anatomical site of satellite cells; insert shows an additional X-MET with evident satellite cells-like (black arrows).

(f) Immunofluorescence analysis performed on whole mount X-MET, cultured for 10 days, for Pax-7 expression and laminin. The image reveals the presence of a satellite cell (red) surrounded by basal lamina positive for laminin (green). Scale bar, $100 \mu \mathrm{m}$. Insert shows a higher magnification of Pax-7 positive cell. Scale bar, $10 \mu \mathrm{m}$. (g) Cluster of myogenic positive cells on the X-MET surface. Scale bar, $10 \mu \mathrm{m}$. (h) X-gal staining of whole mount X-MET, after 10 days in culture. The presence of satellite cells was confirmed by Des/nls-LacZ transgene activation by X-gal staining. Scale bar, $100 \mu \mathrm{m}$.

3D X-MET structure more significantly sustained the up-regulation of vasculogenic and angiogenic factors compared to the $2 \mathrm{D}$ cellular monolayer (Fig. 3d).

One of the advantages to generate a vascularized $3 \mathrm{D}$ structure is that cell survival can be guaranteed after in vivo implantation. At first, we tested this hypothesis in vitro, maintaining X-MET in culture in differentiation medium for 5, 10 and 40 days, analyzing the $\mathrm{X}$ MET morphological features over time and comparing its cell survival with that of the conventional $2 \mathrm{D}$ cell culture muscle model.
Interestingly, while $2 \mathrm{D}$ cell culture system did not survive more than 15 days in DM, X-MET structure was maintained viable in culture for over 40 days (data not shown). To analyze potential morphological changes of the X-MET in culture over time, we performed histological and morphometric analysis on it at 5day, 10-day and 40 days in DM. We did not observe significant differences in the X-MET myotube size between 5 days and 10 days in DM (Fig. 3e), as well as we did not observe a significant difference of the general size and the myotube length between $2 \mathrm{D}$ 
a

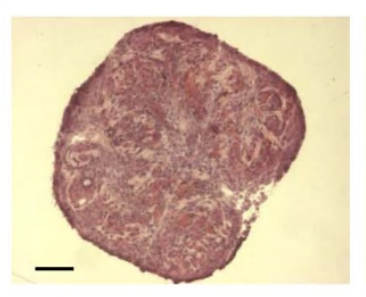

C

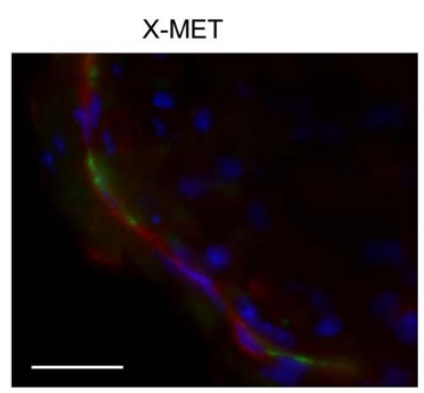

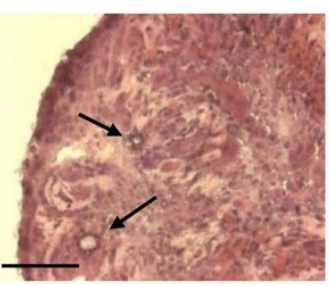

b

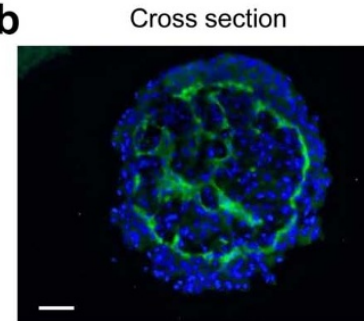

Longitudinal section

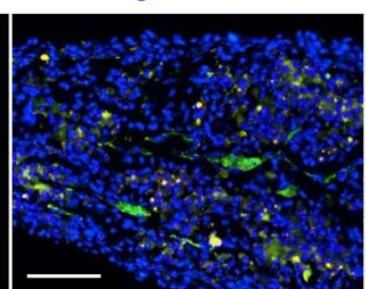

Skeletal muscle

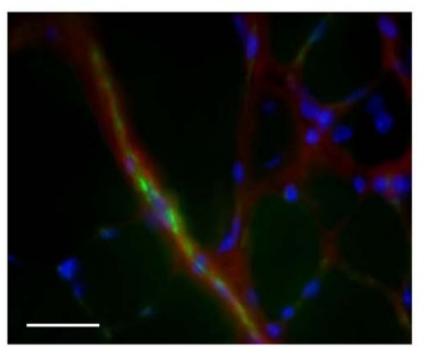

d

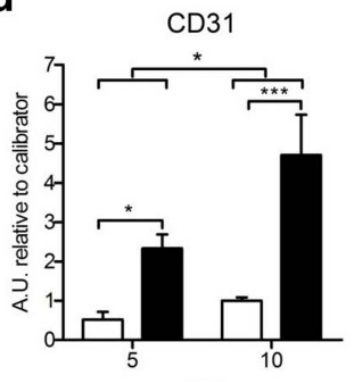

days

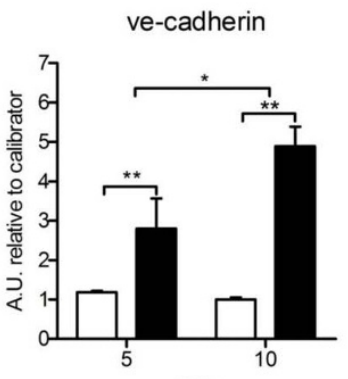

days

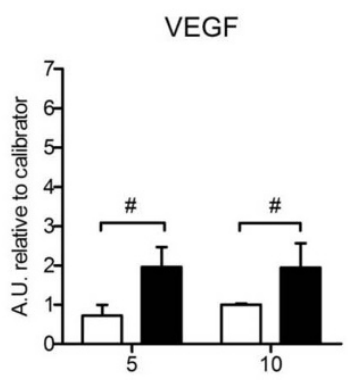

days

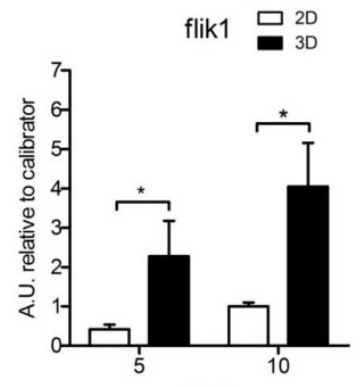

days

e

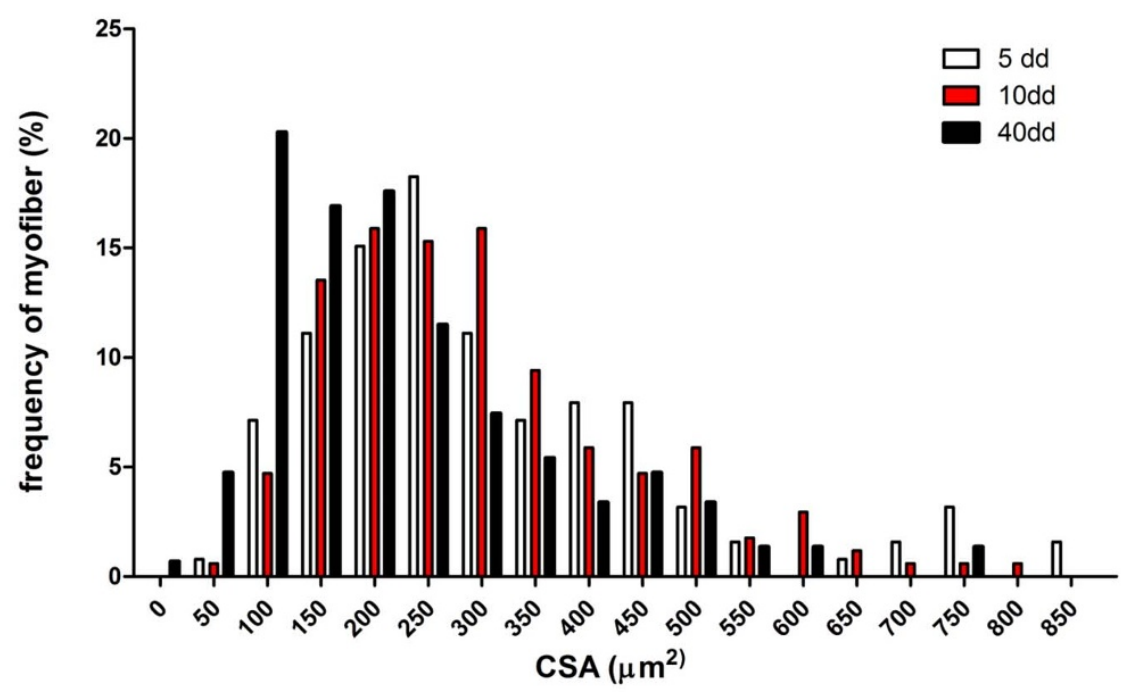

Figure 3 Expression of endothelial markers and vessel-like structures guarantee survival of X-MET. (a) Hematoxylin and eosin staining on cross sections of 10 days cultured X-MET revealed the presence of lumen-like structures (black arrows). Scale bar, $100 \mu \mathrm{m}$. (b) In vitro vascularization of engineered skeletal muscle tissue. X-MET sections were immunostained with anti-CD31 antibodies (green) showing endothelial structures throughout the 3D constructs: the cross section shows the organization of endothelial cells forming a perimeter ring with interior rays which permeate the 3D structure of X-MET, while the longitudinal section shows the presence of endothelial structures along the myotubes. Scale bar, $100 \mu \mathrm{m}$. (c) Immunofiuorescence analysis of X-MET and skeletal muscle cross sections for anti-CD31 (green) and smooth muscle actin (red) expression. Hoechst was used for nuclear staining (blue). X-MET merge image shows the contiguous signals of smooth muscle cells (red) and endothelial cells (green). The organization and subcellular localization of CD31 and smooth muscle actin in the X-MET is similar to that observed in skeletal muscle. Scale bar, $25 \mu \mathrm{m}$. (d) Relative quantitative real time PCR analysis for the expression of endothelial markers between cells cultured in monolayer (2D) and X-METs (3D) after 5 and 10 days in culture. The values represent mean \pm SD of three independent experiments, each obtained pooling 5 samples together. ${ }^{*} \mathrm{p}<0.05$, ${ }^{* *} \mathrm{p}<0.01,{ }^{* * *} \mathrm{p}<0.005$, ${ }^{*} \mathrm{p}=0.06$ (2-way ANOVA test, Bonferroni post hoc-test). (e) Histograms show morphometric analysis. Fiber size for X-MET of 5-day (white bars), X-MET of 10-day (red bars) and X-MET of 40-day (black bars) (mean \pm SEM); X-MET of 5 -day $=319.5 \pm 16.8 \mu \mathrm{m}^{2}$; X-MET of 10 -day $=303.6 \pm 11.5 \mu \mathrm{m}^{2}$; X-MET of 40 -day $=231.0 \pm 11.7 \mu \mathrm{m}^{2}$. The difference in the median values between the X-MET of 40-day and the other time points is statistically significant, Mann-Whitney Rank Sum test: $\mathrm{p}<0.001$. 
and 3D X-MET structure at 5 days and 10 days in DM (data not shown).

Of note, a median value shift toward the small size has been observed for the X-MET after 40-days in culture (Fig. 3e). All of these data suggest that: i) the 3D X-MET structure survives in culture for an extended period of time if compared to the classical 2D structure; ii) The X-MET after 40-days in culture might represent an ideal in vitro muscle model of atrophy-associated senescence and aging, typical of sarcopenia, the age-related loss of skeletal muscle mass $^{24}$.

Measurement of the X-MET biomechanical properties. Three days after formation, X-MET displayed constant and rhythmic spontaneous contractions, which remained unaltered as long as the $\mathrm{X}$-MET remained in culture. The average contraction frequency was $1.5 \mathrm{~Hz}$, and the peak to peak force amplitude ranged from 30 to $90 \mu \mathrm{N}$ (Fig. 4a). We also measured and compared the functional properties of X-MET with that of two different excised muscles that have a similar size, namely Soleus and extensor digitorum longus (EDL). Mouse Soleus muscle is composed primarily of slow type I and type IIA fibers, whereas EDL has predominantly type IIB fibers ${ }^{25}$. When electrically stimulated by means of two platinum electrodes, X-MET showed a contractile response that was consistent with that of adult skeletal muscles: single pulses evoke twitch contraction, while trains of pulses elicit tetanic contractions which produce forces that increase when the frequency increases (Fig. 4b). Functional analysis revealed that X-MET behaves more closely to soleus muscle, although X-METs mean specific force $\left(\mathrm{F}_{\text {spec }}\right)$ was considerably lower: X-MET $F_{\text {spec }}=893 \pm 110 \mu \mathrm{N} / \mathrm{mm}^{2}$; Soleo $\mathrm{F}_{\text {spec }}=198 \pm 6 \mathrm{mN} / \mathrm{mm}^{2}$, EDL $\mathrm{F}_{\text {spec }}=142 \pm 11 \mathrm{mN} / \mathrm{mm}^{2}$ (mean \pm SEM) (Fig. 4b). The dynamic properties of the X-MET isometric twitches were: time to peak (TTP) $=89.4 \pm 3.4 \mathrm{~ms}$ and half relaxation time $(1 / 2 \mathrm{RT})=72.1 \pm 10.2 \mathrm{~ms}($ mean $\pm \mathrm{SEM})$. Again, dynamic properties of X-MET were more similar to Soleus properties $(\mathrm{TTP}=32.0 \pm 1.3 \mathrm{~ms}, 1 / 2 \mathrm{RT}=40.3 \pm 3.0 \mathrm{~ms})$ than to EDL ones $(\mathrm{TTP}=13.9 \pm 0.4 \mathrm{~ms}, 1 / 2 \mathrm{RT}=12.7 \pm 0.5 \mathrm{~ms})$.

Transplanted X-MET shows histological continuity with endogenous muscle fibers. To verify whether X-MET is able to survive and to structurally interact in vivo with pre-existing muscle fibers, we used the whole muscle graft model ${ }^{26-29}$ in which the transgenic XMET, generated from MLC/hAP transgenic mice, was grafted into the tibialis anterior (TA) muscle. MLC/hAP mouse expresses the reporter gene human alkaline phosphatase (hAP) under the transcriptional control of myosin light chain (MLC) promoter. This promoter is selectively activated in differentiated muscle fibers $^{30}$, whereas it is silent in undifferentiated muscle and in nonmuscle cell types. Thus, the hAP is a useful and valuable marker to follow the fate of transplanted cells. We traced donor-derived MLC/ hAP X-MET by hAP stain and by immunofluorescence analysis for the embryonic isoform of Myosin Heavy Chain (e-MyHC), which is normally expressed by in vitro cultured myogenic cells and by regenerating myofibers, but not by adult muscle fibers ${ }^{1}$. Two weeks after grafting X-MET into the injury site (the "bed" in TA), the whole TA muscle was removed and analyzed at histological level. Figure $5 \mathrm{a}$ shows the area of transplant in which the myofibers derived from the transgenic X-MET are clearly identified by the blue-violet coloration of alkaline phosphatase staining (Fig. 5a, left panel) and by e-MyHC expression (Fig. 5a, middle panel). Of note, the hAP positive cells resulted also positive for the expression of e-MyHC (Fig. 5a, middle panel). In addition, immunofluorescence analysis for laminin showed the histological continuity between the donor X-MET and the host muscle fibers (Fig. 5a, right panel). Interestingly, immunofluorescence analysis for CD31 (Fig. 5b) shows the organization of endothelial cells into vessel-like structures in the X-MET graft. a

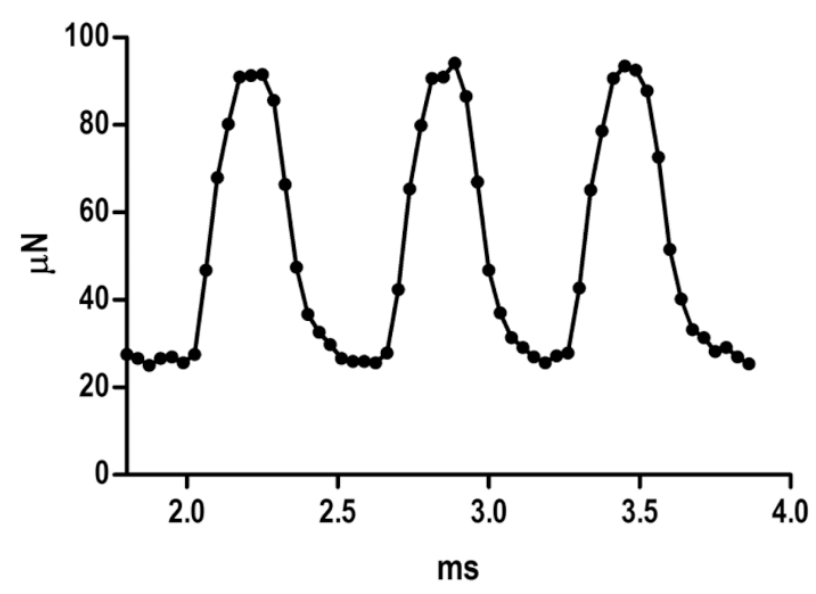

b

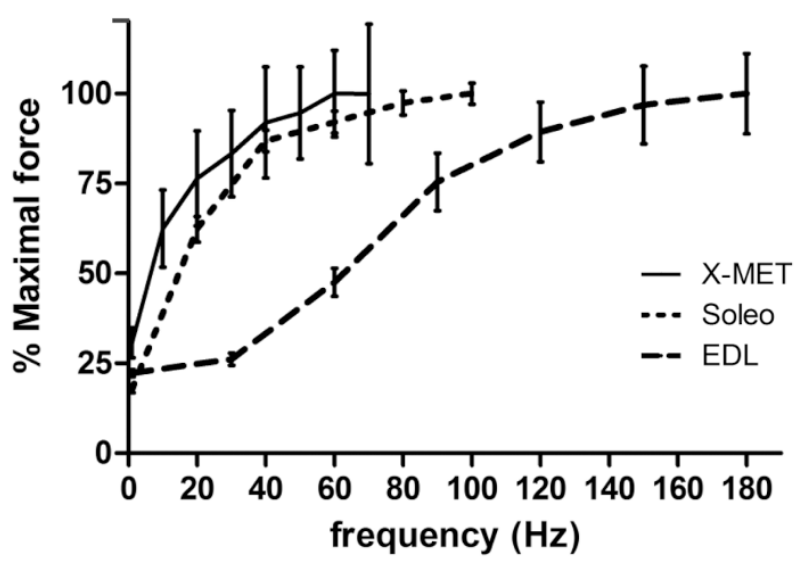

Figure $4 \mid$ Functional analysis. (a) Graph showing spontaneous contraction of X-MET. (b) Force-frequency curves of X-MET $(\mathrm{n}=8)$ compared to control skeletal muscles: EDL $(n=8)$ and Soleus $(n=8)$ muscles. Forces generated by incremental stimulation frequencies were normalized by maximal force. Eight pulse trains of frequencies ranging from 10 to $70 \mathrm{~Hz}$ were delivered to the X-MET to obtain the forcefrequency curve; tetanic force increased with increasing frequency up to $70 \mathrm{~Hz}$, but, on average, the asymptotic value is achieved at $60 \mathrm{~Hz}$.

Transplanted X-MET rescues the ablation of EDL muscle. To verify whether X-MET could behave as the endogenous muscle, we excised the extensor digitorum longus (EDL) muscle of the wild type animal and replaced it with X-MET (Fig. 6a-b-c). Contralateral limb, in which the excised EDL was not replaced by an X-MET, served as control. Interestingly, the limb of mouse transplanted with X-MET was able to flex the toes and to catch an object (Fig. 6d) (Supplementary Video 2), while the contralateral limb was completely unable to do it (Fig. 6e) (Supplementary Video 3).

In order to evaluate the extent of functional recovery with the XMET transplantation we analyzed and compared, by grip test, the skeletal muscle force in presence or absence of X-MET as well as of an uninjured muscle. Of note, in the absence of X-MET, the capacity of generating force was dramatically reduced (Fig. 6f). In contrast, the mouse transplanted with X-MET was able to recover a $14 \%$ of the original force (Fig. 6f). This suggests that, although the mean specific force of isolated X-METs $\left(\mathrm{F}_{\text {spec }}\right)$ is considerably lower than that of endogenous muscles, the X-MET makes the transplanted muscle able to generate significantly more force, compared to untransplanted muscle. 


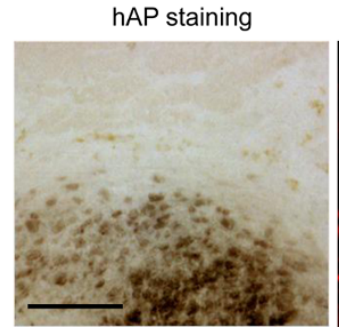

e-MyHC

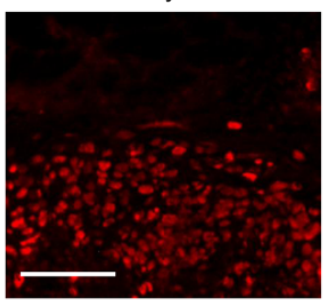

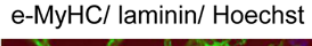

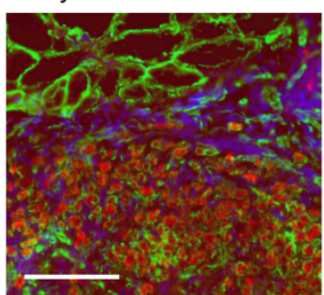

b

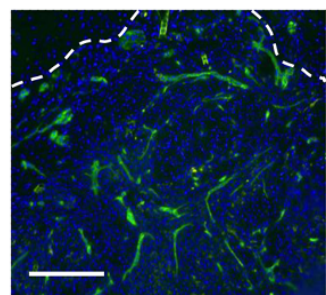

Figure $5 \mid$ In vivo analysis of transplanted X-MET engineered muscle construct. (a) Histochemical and immunohistological analysis of a transversal section of the transplanted area. Left panel: hAP stain (blue-violet coloration) shows fibers of transplanted X-MET. Middle panel: immunofluorescence analysis for embryonic myosin heavy chain (e-MyHC). hAP positive cells express also e-MyHC, revealing the donor origin of these cells. Right panel: immunofluorescence analysis for e-MyHC (red), laminin (green), and nuclei (blue). X-MET shows a histological continuity with the larger myofibers of recipient origin (at the top of panel). Scale bar, $100 \mu \mathrm{m}$. (b) Immunofluorescence for CD31 expression reveals the presence of endothelial structures within the area were X-MET was transplanted. Hoechst was used to stain nuclei (blue). Scale bar, $100 \mu \mathrm{m}$.

\section{Discussion}

The 3D X-MET structures mimicked the structure and function of muscle more closely than classical 2D approaches.

The necessary components for the generation of a $3 \mathrm{D}$ engineered tissue normally include a biodegradable material that serves as a scaffold to facilitate new tissue growth and the differentiation of cell populations within the scaffold.

Our work focused primarily on creating a functional engineered $3 \mathrm{D}$ vascularized skeletal muscle tissue, with the potential capacity to replace muscle tissue lost following severe trauma or diseases. XMET is generated in vitro from muscle primary cultures. At first, we observed that the heterogeneity of muscle isolated cells, such as myoblasts, fibroblasts and endothelial cells are a critical prerequisite to guarantee the cellular complexity typical of in vivo muscle tissue. Indeed, our culture conditions mimic the cell-cell and cell-matrix interactions, necessary for a proper muscle function and activity. We demonstrated that X-MET is a muscle-like structure, mainly composed of differentiated myotubes, expressing myosin, and with a small percentage of undifferentiated cells, expressing relevant markers of activated and committed satellite cells such as Pax-7, desmin, MyoD and myogenin. We also demonstrated that satellite-like cells in the X-MET are surrounded by the basal lamina. This aspect is a

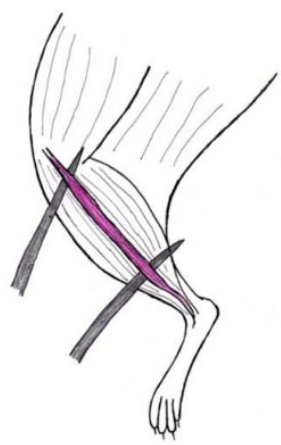

d

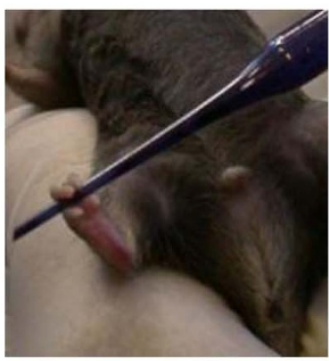

b

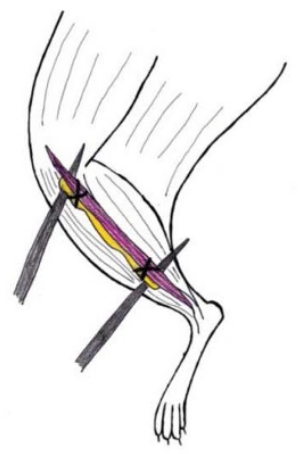

e

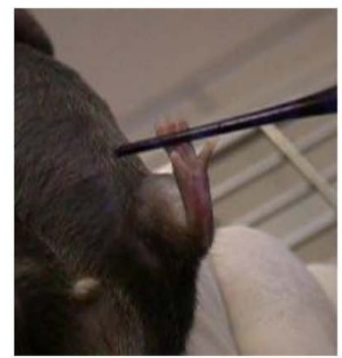

C

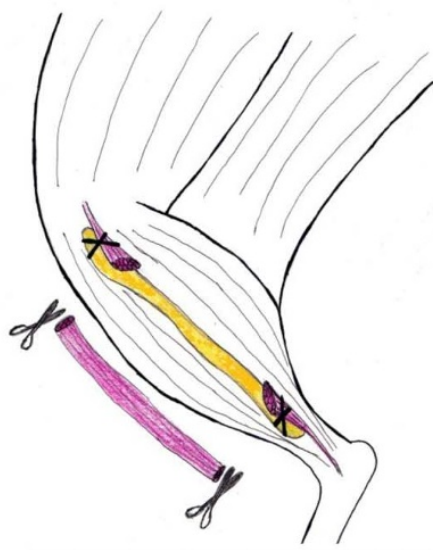

f

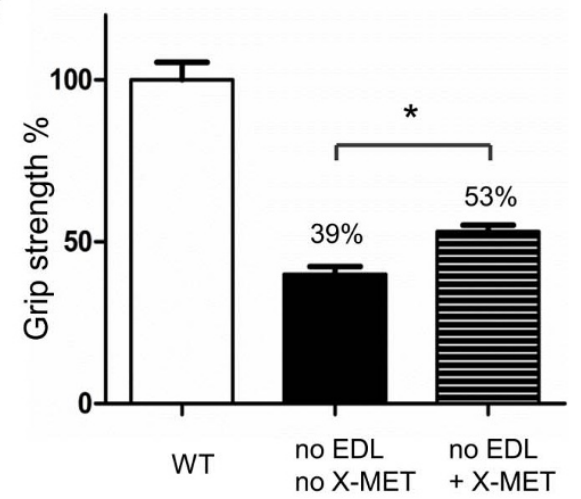

Figure 6 Transplanted X-MET rescue the functional properties of excised EDL muscle. (a-b-c) Schematic illustration of the grafting. (a) The extensor digitorum longus (EDL) was exposed. (b) The ends of the X-MET were sutured on the host EDL. (c) The EDL was transacted and excised. $(\mathrm{d}-\mathrm{e})$ Functional evaluation of transplanted X-MET $(\mathrm{n}=6)$. (d) The transplanted limb, in which the excised EDL muscle was replaced with X-MET, was able to catch an object (Pasteur pipette). (e) The contralateral limb was unable to do it. (f) Histograms show the results of the grip test; the X-MET transplanted mice display a $14 \%$ higher force than the peak force of the mice without X-MET $\left({ }^{*} \mathrm{p}<0.05\right.$, non parametric, Kruskal-Wallis ANOVA test). 
interesting, as it would indicate that in the X-MET structure the satellite cell niche can be reconstructed in vitro.

The high expression levels of endothelial markers, such as CD31, VE-cadherin, vascular endothelial growth factor (VEGF) and its receptor flik1, indicates the formation of some form of in vitro vasculature. These results indicate that the X-MET authentically represents a skeletal muscle-like structure capable of forming prevascularization which is ideal for inducing vascular development within the defected muscle after implantation. This is of vital importance, since an ongoing vascularization process is a crucial requirement for the survival of the $\mathrm{X}-\mathrm{MET}$ in vitro and in vivo. The increased levels of VEGF was associated with the maintenance of X-MET in culture for an extended period of time, compared to the $2 \mathrm{D}$ culture system, suggesting that vascularized X-MET maximizes cell survival, which in turn might maximize the efficacy of in vivo transplantation. In fact, long-term survival of reconstructed skeletal muscle is dependent on good vascularization ${ }^{31,32}$.

In vivo, the nutrients essential for cells to function are provided by the vasculature. However, blood supply is not available for most tissue engineered constructs either in vitro or during the immediate post-implantation phases in vivo ${ }^{33}$. Quite often viable tissue formation is observed in the peripheral regions of a $3 \mathrm{D}$ engineered tissues obtained using synthetic scaffolds, whereas the interior fails to support viable tissue due to the lack of adequate diffusion. This may be a manifestation of the fact that as cells within the pores of the scaffold begin to proliferate and secrete extracellular matrix (ECM), they simultaneously occlude the pores and decrease the supply of nutrients to the interior ${ }^{33}$. In addition, inclusion of a scaffold composed of excess non-contractile matrix would inhibit the formation of myotubes and decrease the specific force and therefore the tissue functionality. Materials with mechanical properties closely approximating those of natural tissue are desirable ${ }^{34}$.

In the X-MET, due to the fact that no artificial scaffold has been used, an adequate diffusion of nutrients should be obtained, with the result of a much longer survival of the 3-D structure in culture.

When electrically stimulated, the X-MET showed a behaviour that is comparable with that of adult skeletal muscles. Thus, X-MET is a muscle-like structure with regard to contractile function as well as morphology and excitability.

To demonstrate the potential clinical application of X-MET, we performed in vivo transplantation, demonstrating the capacity of $\mathrm{X}$ MET to successfully integrate with the host skeletal muscle fibers and to functionally replace the excised EDL muscle.

In summary, our study clearly demonstrates that X-MET is an in vitro engineered muscle structure that closely mimics the complex morphological and functional properties of skeletal muscle tissue, and that it is an ideal tool for regenerative medicine. Moreover, XMET may be considered a useful experimental tool for in vitro studies to characterize molecular mechanisms involved in the maintenance of muscle phenotype, to define the molecular signature of the physiopathological changes over time in culture, to test drugs and, therefore, to limit the use of live animals.

\section{Methods}

Animals. Wild type and transgenic animals ${ }^{35,36}$ used to generate X-MET Animals were housed in a temperature-controlled $\left(22^{\circ} \mathrm{C}\right)$ room with a $12: 12 \mathrm{hr}$ light-dark cycle. Mice were maintained according to the institutional guidelines of the animal facilities of National Institute of Health-Italy.

Primary cultures and generation of X-MET. Mice were sacrificed with cervical dislocation. Primary cultures were obtained using muscle tissue harvested from the hind limbs of wild type (WT), MLC-hAP and desmin nls lacZ transgenic mice (14-18 weeks of age $)^{37}$. The isolated muscles were dissociated both mechanically, by mincing them into a coarse slurry with scissors, and enzymatically, using two different solutions: collagenase/dispase (Roche) $1 \mathrm{mg} / \mathrm{ml}$ and subsequently collagenase type II (Sigma) $0.1 \mathrm{mg} / \mathrm{ml}$, detailed in Musarò and Barberi ${ }^{37}$.

To obtain the correct composition of different cell types, the mixed population of cells was then enriched for myoblasts, using the preplating technique ${ }^{37}$.
Cells were then grown on tissue culture plastic dishes that had been coated with collagen-type I (Sigma) and maintained in GM. At about 5-6 days, when the myoblasts showed an elongated phenotype, the myogenic cells were induced to differentiate by reducing the percentage of HS (Differentiation Medium: DMEM, 5\% horse serum, $25 \mathrm{mM}$ HEPES, $4 \mathrm{mM}$ L-glutamine, 0,1\% gentamicin, penicillin/streptomycin).

After about 2 days in the differentiation medium, the cell monolayer resulted composed of muscle and non-muscle cells, densely packed within a layer of extracellular matrix. Such a monolayer adheres weakly to the culture dish, so that it is easy to detach. We stimulated the monolayer delamination by gently moving a sterile tip around the peripheral area of the plate.

The delaminated monolayer was then pinned on a silicone-coated dish (Sylgard, Dow Corning, Midland, Mich.) using $0.10 \mathrm{~mm}$ diameter stainless steel minutien pins In about 2-3 days the monolayer turned into a self-organized cylindrical structure containing beating myotubes. X-METs grew, on average, to a diameter of $197 \pm 12 \mu \mathrm{m}$ and a length of $9 \pm 2 \mathrm{~mm}$.

Flow cytometry. Adherent muscle-derived cells were analysed using a Coulter Epics XL flow cytometer (Beckman Coulter Fullerton, CA, USA). The following antibodies for cell staining were used: anti CD45 eFluor 450; anti CD31 PE-Cy7 (eBioscience) anti-Sca1-FITC (BD Pharmingen), anti- $\alpha 7$-integrin-PE (MBL International Corporations). All suspensions were stained with SYTOX ${ }^{\circledR}$ Blue to exclude dying or dead cells. For each analysis, data were collected from 30,000 cells and analyzed using Win MDI 2.8 software (Scipps Research Institute, La Jolla, CA).

Histological and immunofluorescence analysis. X-METs were embedded in tissue freezing medium and snap frozen in nitrogen-cooled isopentane. Samples were mounted on a cryostat and cut at $7 \mu \mathrm{m}$ thick sections. Frozen sections were stained for hematoxylin \& eosin and X-gal ${ }^{36}$.

For immunofluorescence analysis, samples were fixed with 4\% PFA and incubated overnight at $4{ }^{\circ} \mathrm{C}$ with primary antibodies: MyoD (BD Biosciences Pharmingen), myogenin (Dako), MyHC (MF20 Hybridoma Bank), laminin (Sigma), ERTR7 (Acris), CD31 (Abcam), $\alpha$-SMA (Sigma), Pax-7 (Hybridoma Bank), e-MyHC (Hybridoma Bank); nuclei were visualized using Hoechst staining. Samples were visualized using an inverted microscope (Axioskop 2 plus; Carl Zeiss MicroImaging, Inc.); images were processed using Axiovision 3.1.

RNA extraction and real time PCR analysis. Total RNA extraction was performed using TriRiagentTM (SIGMA) and was reverse-transcribed using the QuantiTec Reverse Transcription kit (QIAGEN). Relative quantitative PCR was performed on ABI PRISM 7500 SDS (Applied Biosystems, USA), using premade 6carboxyfluorescein (FAM)-labeled TaqMan assays for HPRT1, MyoD, Myogenin, Pax-7, CD31, VE-cadherin, Flik1, VEGFA (Applied Biosystem, USA). Relative quantitative RT-PCR sample value was normalized for the expression of HPRT1mRNA. The relative level for each gene was calculated using the 2-ddCt method $^{38}$ and reported as mean fold change in gene expression.

Morphometric analysis and statistics. The size of $\mathrm{MyHC}$ positive myotubes was calculated using Scion Image software (v. Beta 4.0.2, Scion, Frederick, MD). Statistical analysis was performed with GraphPad Prism v5.0 software; groups were compared by Mann-Whitney Rank Sum test and the difference in the median values between the two groups was considered significant for $\mathrm{p}$ value $<0.05$.

X-MET transplantation in skeletal muscle. Muscle Graft model 1: Transplanting $\mathrm{X}$-MET into injured muscle.

C57BL/6 wild type mice were anesthetized with an intraperitoneal injection of $250 \mathrm{mg} / \mathrm{Kg}$ of Tribromoethanol (Avertin ${ }^{\circledR}$ ). The skin on the hind limbs was opened, and the tibialis anterior (TA) muscles were gently exposed. In host TA muscles, we created a local injury (a "bed") in the middle region of the muscle by removing a small number of fibers. The bed was 5-7 $\mathrm{mm}$ long and $3 \mathrm{~mm}$ in deep. We transplanted an $\mathrm{X}$-MET fragment, obtained from MLC/hAP mice, into the bed obtained in the right TA muscle. The length of the X-MET fragment was adapted to perfectly fit the bed.

Before the transplant the MLC/hAP X-MET fragment was washed twice in PBS and then sutured on the host muscle (5/0 silk). The removal of skeletal muscle fibers from left TA served as control experiments. 15 days following the transplant, both TAs were carefully removed in their entirety, snap frozen and sectioned for histological analysis.

Muscle graft model 2: Repair of transected muscle with X-MET and grip test. C57BL/6 wild type mice were anesthetized and the EDL muscle was exposed. MLC/ hAP X-MET was washed twice in PBS. The ends of the X-MET were sutured on the host EDL at a distance of 1-2 mm from its tendons, following which the EDL was transected in the middle region. The X-MET remained in the site previously occupied by the EDL muscle, with its ends sutured to the remains of EDL tendons. The X-MET was then enclosed by suturing the overlying tibialis anterior muscle and skin with 5/0 silk. Control experiments included removal of EDL from the contralateral limb without any muscle substitute.

After 15 days the functionality of the transplanted limbs was evaluated by grip test. Groups of 6 mice were analyzed twice on two different days each time in three repetitions. The grip-strength apparatus (47105/47106 Ugo Basile) was comprised of a T-shaped grasping bar connected to a force transducer connected to the Peak Amplifier 47105-001. The animal is lowered toward the platform and gently pulled backwards with consistent force by the experimenter until it releases its grip, allowing 
assessing muscular strength in limb muscles. The grip force is recorded on the strain gauge. The mean of three measurements was calculated for each animal.

X-MET mechanical measurements. The X-MET was placed in a culture dish containing a calcium free solution (Krebs Ringer Bicarbonate Buffer) at controlled temperature $\left(37^{\circ} \mathrm{C}\right)$. One end of the X-MET was linked to a force transducer, and the other was connected to a micromanipulator (Burleigh, 6000 ULN) used to precisely adjust the X-MET length.

The force transducer (Kronex Tech Co, AE 801) was equipped with a hook made of Stainless Steel Wire (Kronex Tech Co, $0.12 \mathrm{~mm}$ diameter) to allow the attachment of the X-MET. Electrical pulses generated by a pulse stimulator (Aurora Scientific Inc. 701B) were delivered to the X-MET through two platinum electrodes placed paralle to the tissue. The stimulation pulses were square and unipolar. A control program, developed in LabView2011, allows to set pulses parameters (duration, amplitude and frequency) and the duration of each train of pulses.

The X-MET diameter was measured using a microscope slide with a precision rectangular-coordinate grid pattern with a resolution of $0.1 \mathrm{~mm}$. The total cross sectional area (CSA) of the X-MET was then calculated assuming a circular section for the tissue.

Current intensity and pulse duration were set to $400 \mathrm{~mA}$ and $1 \mathrm{~ms}$ respectively, which were shown in preliminary experiments to elicit maximal twitch force $\left(\mathrm{F}_{\mathrm{tw}}\right)$ Before starting each experiment, X-MET length was adjusted by using a micromanipulator to the length $\left(\mathrm{L}_{0}\right)$ that allowed the tissue to elicit the maximum twitch force. A force-frequency diagram was obtained by stimulating the X-METs with a $1.2 \mathrm{~s}$ train of pulses with increasing frequencies up to $70 \mathrm{~Hz}$. The X-MET was allowed to rest for 180 seconds between stimulus trains. Maximum isometric tetanic force $\left(F_{\max }\right)$ was taken as the plateau of the force-frequency curve.

Specific tetanic force $\left(\mathrm{F}_{\text {spec }}\right)$ was obtained by dividing the maximum tetanic force by the X-MET cross sectional area. Time to peak (TTP) was determinated by measuring the time from pulse release until maximum of twitch force $\left(\mathrm{F}_{\mathrm{tw}}\right)$, while time for force relaxation $1 / 2 \mathrm{RT}$ was determined by measuring the time from $\mathrm{F}_{\mathrm{tw}}$ to the time that corresponds to a value of force equal to $1 / 2 F_{t w}$

EDL and Soleus muscles mechanical measurements. EDL and Soleus muscles were isolated from 4-month-old WT mice and electrically stimulated as previously described $^{39}$. In particular EDL specimens were stimulated at 30, 60, 90, 120, 150 and $180 \mathrm{~Hz}$, while Soleus muscles were stimulated at 20, 40, 60, 80 and $100 \mathrm{~Hz}$. Specific force was computed as maximum force divided by muscle CSA ${ }^{39}$.

Statistical analysis. Unless otherwise specified, results were expressed as mean \pm SEM. For relative quantitative real time PCR analysis the values represent mean \pm SD of three independent experiments, each obtained pooling 5 samples together. The differences were considered significant for $\mathrm{p}$ value $<0.05\left({ }^{*} \mathrm{p}<0.05\right.$, ${ }^{*} \mathrm{p}<0.01$, $*_{* *} \mathrm{p}<0.005,{ }^{*} \mathrm{p}=0.06$ ) (2-way ANOVA test, Bonferroni post hoc-test).

1. Carosio, S., Berardinelli, M. G., Aucello, M. \& Musaro, A. Impact of ageing on muscle cell regeneration. Ageing Res. Rev. 10, 35-42 (2011).

2. Vinciguerra, M., Musaro, A. \& Rosenthal, N. Regulation of muscle atrophy in aging and disease. Adv. Exp. Med. Biol. 694, 211-233 (2010).

3. Mooney, D. J. \& Mikos, A. G. Growing new organs. Sci. Am. 280, 60-65 (1999).

4. Bach, A. D. et al. A new approach to tissue engineering of vascularized skeletal muscle. J. Cell Mol. Med. 10, 716-726 (2006).

5. Olson, J. L., Atala, A. \& Yoo, J. J. Tissue engineering: current strategies and future directions. Chonnam. Med. J. 47, 1-13 (2011)

6. Vandenburgh, H. et al. Tissue-engineered skeletal muscle organoids for reversible gene therapy. Hum. Gene Ther. 7, 2195-2200 (1996).

7. Shansky, J., Del Tatto, M., Chromiak, J. \& Vandenburgh, H. A simplified method for tissue engineering skeletal muscle organoids in vitro. In Vitro Cell Dev. Biol. Anim 33, 659-661 (1997).

8. Okano, T. \& Matsuda, T. Hybrid muscular tissues: preparation of skeletal muscle cell-incorporated collagen gels. Cell Transplant 6, 109-118 (1997)

9. Okano, T. \& Matsuda, T. Tissue engineered skeletal muscle: preparation of highly dense, highly oriented hybrid muscular tissues. Cell Transplant 7, 71-82 (1998).

10. van Wachem, P. B., van Luyn, M. J. \& da Costa, M. L. Myoblast seeding in a collagen matrix evaluated in vitro. J. Biomed. Mater. Res. 30, 353-360 (1996).

11. Huang, Y. C., Dennis, R. G., Larkin, L. \& Baar, K. Rapid formation of functional muscle in vitro using fibrin gels. J. Appl. Physiol 98, 706-713 (2005).

12. Dennis, R. G. \& Kosnik, P. E. Excitability and isometric contractile properties of mammalian skeletal muscle constructs engineered in vitro. In Vitro Cell Dev. Biol. Anim 36, 327-335 (2000).

13. Molgo, J., Colasantei, C., Adams, D. S. \& Jaimovich, E. IP3 receptors and Ca2+ signals in adult skeletal muscle satellite cells in situ. Biol. Res. 37, 635-639 (2004).

14. Fukada, S. et al. Purification and cell-surface marker characterization of quiescent satellite cells from murine skeletal muscle by a novel monoclonal antibody. Exp. Cell Res. 296, 245-255 (2004).

15. Jankowski, R. J., Deasy, B. M., Cao, B., Gates, C. \& Huard, J. The role of CD34 expression and cellular fusion in the regeneration capacity of myogenic progenitor cells. J. Cell Sci. 115, 4361-4374 (2002).

16. DeLisser, H. M., Newman, P. J. \& Albelda, S. M. Molecular and functional aspects of PECAM-1/CD31. Immunol. Today 15, 490-495 (1994).

17. Joe, A. W. et al. Muscle injury activates resident fibro/adipogenic progenitors that facilitate myogenesis. Nat. Cell Biol. 12, 153-163 (2010).
18. Wang, Y. X. \& Rudnicki, M. A. Satellite cells, the engines of muscle repair. Nat. Rev. Mol. Cell Biol. 13, 127-133 (2012).

19. van Vliet, E., Melis, M., Foidart, J. M. \& van Ewijk, W. Reticular fibroblasts in peripheral lymphoid organs identified by a monoclonal antibody. J. Histochem. Cytochem. 34, 883-890 (1986).

20. Mauro, A. Satellite cell of skeletal muscle fibers. J. Biophys. Biochem. Cytol. 9 , 493-495 (1961).

21. Zammit, P. S. et al. Muscle satellite cells adopt divergent fates: a mechanism for self-renewal? J. Cell Biol. 166, 347-357 (2004).

22. Lescaudron, L., Creuzet, S. E., Li, Z., Paulin, D. \& Fontaine-Perus, J. Desmin-lacZ transgene expression and regeneration within skeletal muscle transplants. J. Muscle Res. Cell Motil. 18, 631-641 (1997).

23. Rivron, N. C., Liu, J. J., Rouwkema, J., de Boer, J. \& van Blitterswijk, C. A Engineering vascularised tissues in vitro. Eur. Cell Mater. 15, 27-40 (2008).

24. Evans, W. J. Skeletal muscle loss: cachexia, sarcopenia, and inactivity. Am. J. Clin. Nutr. 91, 1123S-1127S (2010)

25. Augusto, V., Padovani, C. R. \& Compos, G. E. R. Skeletal muscle fiber types in C57BL6J mice. Brazilian Journal of Morphological Sciences 21, 89-94 (2004)

26. Grounds, M. D. et al. Silencing TNFalpha activity by using Remicade or Enbrel blocks inflammation in whole muscle grafts: an in vivo bioassay to assess the efficacy of anti-cytokine drugs in mice. Cell Tissue Res. 320, 509-515 (2005).

27. Roberts, P. \& McGeachie, J. K. In Experimental Models for Transplantation in Small Animals. Vol. 1, Edn. eds. Green, M. K. \& Mandel, T. E. 213-224 (Harwood Academic Publishers, Chur, Switzerland; 1995).

28. Roberts, P., McGeachie, J. K., Grounds, M. D. \& Smith, E. R. Initiation and duration of myogenic precursor cell replication in transplants of intact skeletal muscles: an autoradiographic study in mice. Anat. Rec. 224, 1-6 (1989).

29. Shavlakadze, T., Davies, M., White, J. D. \& Grounds, M. D. Early regeneration of whole skeletal muscle grafts is unaffected by overexpression of IGF-1 in MLC/ mIGF-1 transgenic mice. J. Histochem. Cytochem. 52, 873-883 (2004).

30. Musaro, A. \& Rosenthal, N. Maturation of the myogenic program is induced by postmitotic expression of insulin-like growth factor I. Molecular and Cellular Biology 19, 3115-3124 (1999).

31. Koffler, J. et al. Improved vascular organization enhances functional integration of engineered skeletal muscle grafts. Proc. Natl. Acad. Sci. U. S. A. 108, 14789-14794 (2011).

32. Zhou, W. et al. Angiogenic gene-modified myoblasts promote vascularization during repair of skeletal muscle defects. J. Tissue Eng Regen. Med. (2013).

33. Karande, T. S., Ong, J. L. \& Agrawal, C. M. Diffusion in musculoskeletal tissue engineering scaffolds: design issues related to porosity, permeability, architecture, and nutrient mixing. Ann. Biomed. Eng 32, 1728-1743 (2004).

34. Lutolf, M. P., Gilbert, P. M. \& Blau, H. M. Designing materials to direct stem-cell fate. Nature 462, 433-441 (2009).

35. Grieshammer, U., Sassoon, D. \& Rosenthal, N. A transgene target for positional regulators marks early rostrocaudal specification of myogenic lineages. Cell 69, 79-93 (1992)

36. Musaro, A. et al. Localized Igf-1 transgene expression sustains hypertrophy and regeneration in senescent skeletal muscle. Nature Genetics 27, 195-200 (2001).

37. Musaro, A. \& Barberi, L. Isolation and culture of mouse satellite cells. Methods Mol. Biol. 633, 101-111 (2010).

38. Livak, K. J. \& Schmittgen, T. D. Analysis of relative gene expression data using real-time quantitative PCR and the 2(-Delta Delta C(T)) Method. Methods 25, 402-408 (2001).

39. Del Prete, Z., Musaro, A. \& Rizzuto, E. Measuring mechanical properties, including isotonic fatigue, of fast and slow MLC/mIgf- 1 transgenic skeletal muscle. Annals of Biomedical Engineering 36, 1281-1290 (2008).

\section{Acknowledgments}

This work was supported by 7FP-Myoage (grant N. 223576) and AFM, and partly by Fondazione Roma, Fondazione Thierry Latran, PRIN and Ateneo. The authors would like to thank other members of the Musaro laboratory for helpful discussions and Dr Peter Grigg for his critical comments and his English revision.

\section{Author contributions}

S.C. and L.B. generated the X-MET and performed histological and molecular analysis; S.C., E.R., Z.DP. performed functional analysis and interpreted the results; C.N. performed in vivo transplantation experiments; A.M. designed the study, organized the experiments, interpreted the results, and wrote the paper.

\section{Additional information}

Supplementary information accompanies this paper at http://www.nature.com/ scientificreports

Competing financial interests: The authors declare no competing financial interests.

License: This work is licensed under a Creative Commons

Attribution-NonCommercial-NoDerivs 3.0 Unported License. To view a copy of this license, visit http://creativecommons.org/licenses/by-nc-nd/3.0/

How to cite this article: Carosio, S. et al. Generation of eX vivo-vascularized Muscle Engineered Tissue (X-MET). Sci. Rep. 3, 1420; DOI:10.1038/srep01420 (2013). 